\title{
Supervised machine leaning based anomaly detection and diagnosis in grid connected photovoltaic systems
}

\author{
Swetha R Kumar ${ }^{1}$, Phavithraa Devi B ${ }^{2}$, Gayathri R G ${ }^{3}$ \\ \{srk.ice@psgtech.ac.in ${ }^{1}, 17 u 232 @$ psgtech.ac.in ${ }^{2}, 17 u 213 @$ psgtech.ac.in address ${ }^{3}$ \} \\ Department of Instrumentation and control systems engineering, PSG College of Technology, \\ Coimbatore, Tamil Nadu, India
}

\begin{abstract}
Over the past few decades, due to the slackening in the available nonrenewable resources and the paramount of pollutants caused by the consumption of these non- renewable resources, renewable energy has gained huge momentousness in power generation. This enlarged the installation of Solar Power plants. But these Solar based Photovoltaic systems are liable to a huge variety of anomalies which generally deteriorates its performance. If those anomalies were not identified on the dot, this may lead to the reduction in efficiency which makes the PV systems unsubstantial. Hence the diagnosis of the anomaly and the identification of its type is of whopping importance to maintain the ideal function of the PV array system. This paper aims to predict and classify the types of anomalies in Photovoltaic Array systems using supervised machine learning algorithms. The performance of the classifier based on Naive Bayes, One Vs Rest and Multi-layer Perceptron Neural Network model on the provided dataset is tested. The results thus obtained from training and testing the models, are also tabulated and the observations are noted. Simulated results disclosed that the Multi- Layer perceptronbased classifier has the utmost accuracy with minimum mean squared error.
\end{abstract}

Keywords: Fault detection, PV Array System, Naive Bayes, One Vs Rest Classifier, Multi-Layer Perceptron, supervised learning.

\section{Introduction}

Photovoltaic systems are susceptible to a number of anomalies that should be identified as early as possible before any deviations from the nominal circumstances are observed. To avoid slight deviations from normal conditions and to match the projected energy production and desired power quality, early detection of potential anomalies in PV systems is crucial to effective performance. As an outcome, preventative measures must be implemented to avoid a decline in performance which significantly reduces efficiency, dependability, and safety.

The problem mentioned has already been explored and a number of possible solutions have been provided. Approaches like probabilistic neural network classifier [1], decision trees [2], random forest and neural networks classifiers [3,4] were proposed in literature. Though these approaches has a number of advantages, their shortcomings must also be noted.

In probabilistic neural network classifier, PV module short circuits are not detectable [1]. The key benefits of employing decision trees are the quick learning and classification steps, as well as the ease with which an algorithm or software may be implemented. Even though it has a greater accuracy, it has several drawbacks, such as a high training cost and the possibility that the model will not operate effectively when dealing with unknown input that is not part of 
the training set. [2]. Neural networks techniques provide the best validation performance because they provide the highest accuracy. It is generally used to detect anomalies because it is capable of handling complex data with both linear and non-linear fluctuations. [3].

\section{Photovoltaic System}

Solar cells are the individual cells that make up photovoltaic (PV) panels. Each solar cell produces a modest quantity of electricity. A solar panel is formed when many solar cells are linked together to produce a large amount of electricity. PV systems come in a variety of sizes, depending on the application. It can range from modest rooftop or building-integrated systems with tens of kilowatts of capacity to giant utility-based stations that generate massive amounts of electricity. PV systems that are connected to the electrical grid and systems that allow users to unplug from the grid are both available.

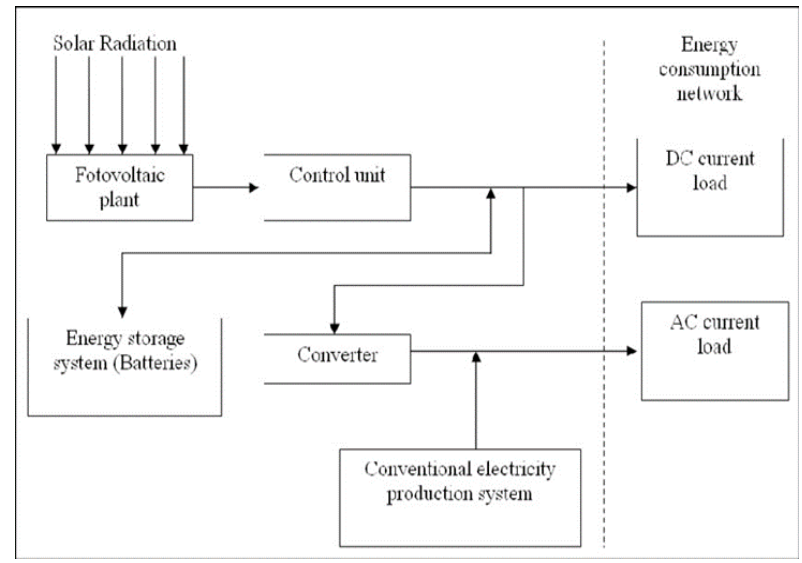

Fig. 1. General layout of PV plant [7]

The conversion of photons to electrons provides DC electricity in solar cells. It can be used to charge batteries and power a variety of equipment, but it must be converted to AC power for usage in the home. The electrical grid transmits power across large distances using $\mathrm{AC}$ power. Certain equipment may run on AC power while others demand DC power, therefore the AC power can be converted to DC once it reaches the end user if required.

The majority of today's PV systems allow consumers to increase or decrease power capacity at any moment. As demand fluctuates, this mechanism allows them to alter their power capacity. Aside from the solar panels, PV systems have many other elements such as inverters, batteries, mechanical installation components, relays, and chargers. These elements aid in the efficient and safe storage and distribution of power.

\section{Faults in photoVoltaic systems}

Anomaly detection in solar photovoltaic systems is a critical task for improving the system's efficiency, dependability, and safety. If the flaws are not identified, not only will power generation be reduced, but the entire system's availability would be jeopardized. PV systems are susceptible to a variety of defects; therefore, before implementing monitoring and fault detection methods, it is vital to determine the sorts of failures that can be detected in the real system.

The six types of faults [5] which are considered for this project are;

- "Partial shading fault" 
- "Bypass diode fault"

- "Bridging fault"

- "Temperature fault"

- "Complete shading fault"

- "Short circuit fault"

Partial shading fault

A photovoltaic system is highly vulnerable to partial shading. It is a case when the different modules of the array receive a different irradiance level. This shadow may be either due to different conditions: nearby building, nearby tree or difficult to predict due to clouds or building. It is considered that the maximum power output of a partial shading PV system always drops as the shading severity rises, depending on the functioning of a PV system that relies on solar irradiance to produce electrical energy.

Bypass diode fault

Due to the relevance of bypass diodes in PV modules, the detection of bypass diode failure in PV systems has become a hot topic. Because bypass diodes are parallelized with PV modules, the goal is to limit the maximum reverse current of PV modules that are partially shaded. As a result, in partial shade conditions, bypass diodes in PV modules allow power to be supplied. PV modules commonly use silicon p-n and Schottky bypass diodes. Both have a wide variety of current ratings, however the Schottky diodes have a substantially smaller forward voltage drop of roughly $0.4 \mathrm{~V}$ compared to the $0.7 \mathrm{~V}$ forward voltage drop of the $\mathrm{p}-\mathrm{n}$ diodes.

Other techniques, including that of the cool bypass switch and the bipolar transistorbased bypass technique, aim to improve PV subpanel durability and output electricity production by integrating a more complicated electronics circuit design into the PV module junction-box.

Bridging fault

When a low resistance connection is detected between two sites of different voltage in a string of modules, a bridging error occurs. These failures are caused by cable insulation system failure, such as an animal chewing through the insulation, water intrusion or corrosion, or mechanical damage. This usually results in a lower open circuit voltage, but a much smaller reduction in short circuit current.

Temperature fault

The temperature is one of most crucial parameters that impact the PV system's power production. When the irradiance is kept constant, the temperature rises, resulting in a lower open circuit voltage. The short circuit current, on the other hand, stays constant.

Complete shading fault

The total shading anomaly occurs if all modules in a photovoltaic array get the same solar insolation that is less than $1000 \mathrm{~W} / \mathrm{m} 2$. Under this type of malfunction, the peak point current, voltage, and power collapses.

Short circuit fault

The short circuit fault in a PV module is mainly due to bad wiring in the system Aging, vibration and abrasion are other factors which can also be recognized as the possible causes of such faults. The short circuit current stays unchanged throughout this anomaly, while the open circuit voltage drops fast.

feature selection

Generally, feature selection is a process in which the numbers of input variables are reduced while developing a predictive model. If the features selected are irrelevant to the output variable or if it is partially irrelevant the accuracy of the system will be highly reduced 
and also prediction will be of increased error. Major benefits of performing feature selection are,

1) Reduction in over fitting, because possibilities of training the model with noise is highly reduced.

2) Increase in Accuracy, since there is a reduction in misleading data.

3) Reduction in Training time, since there is a reduction in amount of data and complexity of algorithm.

In this project initially there were thirteen different features. In order to choose the important features, Feature importance, Correlation matrix with heat map and Wrapper methods have been implemented. Important concepts in these methods will be discussed further.

Feature importance

In Feature importance method of feature selection, Scores is assigned to individual features based upon their importance in predicting the classes or the target variable. In order to calculate the score different methods like decision trees, random forest, statistical correlation, permutation and coefficients calculated using linear models can be used. Thus, using such score, we can get a better understanding of both the dataset and the model with reduction in the number of features. Feature importance provides a deep insight about the dataset with the help of the score and the bar graph. Thus, feature with high relevance is easily spotted with the help of the bar graph plotted. Here in order to obtain the important features extra tree classifier and permutation models has been used.

Correlation matrix with heat map

In order to identify the interaction between different features under consideration correlation matrix can be used. Interaction between the models must be known because by including the highly correlated features the model will be highly weaken the model's performance. This is because correlated features will add inaccuracy and noise to the trained model therefore it is a greater hindrance to our desired classifier. In general features in the dataset can be either positively correlated or it can be negatively correlated. Positive correlation means those features will be directly proportional whereas negatively correlated means the features will be indirectly proportional. Therefore, the feature to be chosen must be positively correlated with the output class and it should not be correlated with any other features.

\section{Wrapper method}

In this method of feature selection, the entire process is based on a specific model. This model can be either linear or non-linear. But non-linear models are highly preferred. This method works based on the principle of greedy algorithm. This model performs a greedy search against all the combinations of features against the desired class or the important parameters like accuracy or error. Since, it calculates all the possible combination of features it takes a huge amount of time to get trained.

Fault detection and diagnosis methodology

Fault detection and diagnosis is the core of this project. Next to feature selection this process of classification was carried out with the important features selected and in this project three different classifier named, Naïve Bayes, One Vs Rest and Multilayer perceptron Classifier were implemented in order to detect and diagnose the identified faults. The theoretical concepts related to these multiclass classifiers will be discussed further.

Naive bayes classifier

Naïve Bayes classifier can be either used for binary classification or it can be used for multiclass classification. This classifier works based on the Naïve Bayes algorithm, which 
states that the all features under consideration are initially considered to be completely independent. And each and every feature are considered to be relevant. This means that, each and every feature contributes to the classification, though it is not practically proven to be true, this is considered to be relevant according to the naïve bayes formula. Hence according to Naïve Bayes classifier all the features in the dataset are completely unrelated. Hence one feature will not affect the presence of another feature. Generally, Bayes theorem can be written as,

$$
P(C \mid X)=\frac{P(X \mid C) P(C)}{P(X)}(1)
$$

In Above Equation (1),

1) " $\mathrm{P}(\mathrm{C} \mid \mathrm{X})$ is the final probability, i.e. Posterior probability of the desired class given Predictor (X, attributes)".

2) " $P(C)$ is the Prior or Initial probability of the class".

3) " $\mathrm{P}(\mathrm{X} \mid \mathrm{C})$ is the is the predicted probability of the predictor given class".

4) " $\mathrm{P}(\mathrm{X})$ is the initial probability of the predictor". [8]

Generally Naïve bayes classifier creates a frequency table for each and every class based on features of the training dataset, and then the likelihood table is created for each and every class. Later the posterior probability is calculates using the Naïve Bayes formula given in the above equation (1). Finally, the classifier checks for the calculated Posterior probability and allocates the input to the class with higher probability.In real time Bernoulli, Categorical or the Gaussian model can be used for training the classifier.In this project in order to predict the probability different models like "Gaussian Naïve Bayes Classifier, Multinomial Naïve Bayes classifier and Bernoulli Naive Bayes classifier" has been implemented and the best out of them has been chosen.

One vs rest classifier

One Vs Rest is a special type of classifier in which each and every class will have a specific binary classifier. This One Vs Rest classifier is mostly used for multiclass classification, hence any input given to any classifier will be considered as a binary input i.e., either it will belong to that class or another class. Hence Accuracy will be high in this case on comparison with Naïve Bayes, but the only disadvantage on comparison with Naïve Bayes is its training time. [9] Training time is comparatively high because if the number of class increases number of individual classifiers also increases. Accuracy will be high because it has multiple classifiers for multi class classification. In order to implement this classifier, one must initially determine the binary classifier to be used for this specific dataset. In order to choose the classifier, trial-and-error method can be implemented. Hence for the given data set the following binary classifier were implemented,

1. Gradient Booster

2. Linear SVC

3. Single layer Perceptron

4. Logistic regression 
Because the activation function is so important in determining accuracy, each and every activation were tried to be implemented and the best activation function was chosen from them. Such trial-and-error method helped a lot in improving the model's performance.

Multilayer perceptron

Perceptron is generally very useful in binary classification, because they work well for linearly separable data. But in the case of multiclass classification a single perceptron cannot achieve our desired classification report. Hence in order to obtain a multiclass classification with the help of perceptron one can use Multilayer perceptron, which contains more than one perceptron thus helps in achieving multiclass classification with higher accuracy. In practice, a multilayer perceptron network is made up of three layers: the input layer, hidden layers, and output layer.. Input Layer consists of nodes which receives the input and hidden layers can be of any number depending upon the complexity of the dataset or the classification, and finally output layer process the entire neurons and gives the particular class as of the input dataset as an output.[6]

In the process of training the model, these models propagate through the networks either in forward motion or in the reverse motion in order to adjust the parameters. Only when these parameters are adjusted, the error will turn out to be minimum. Or else without adjusting the parameters like bias, and weights the network will not work efficiently as expected.

Forward propagation and backward propagation are the two different mechanisms through which one can traverse throughout the neural network. In the case of forward propagation, to traverse from the input layer to the output layer through the hidden layers, whereas in the case of backward propagation the entire process is completely reversed. In backward propagation, generally mathematical concepts like, partial derivation or the chain rule in the mathematic chapter calculus, for the error functions with respect to the various weights and the biases in our network are used. Thus, in the backward means the error gets eliminated by adjusting the parameters like bias and weights.[10]

Using these three different multiclass classifiers the faults can be detected and classified. Results and observation of implementing these classifiers will be discussed further.

\section{Results and observation}

The implementation is carried out in Python language. The platform chosen is Colaboratory since colab notebook facilitates writing and executing Python in the browser, without any setup necessary, free GPU usage, and easy sharing. The dataset is obtained from IEEE dataport [11]

Selection of best features

Here is the obtained output after simulating different feature selection methods like Feature importance, Correlation matrix with heat map, and Wrapper method. With the help of the results obtained from these feature selection methods the dataset can be modified according to these important features and then in can be applied for the process of training and testing.

There might be slight variations in the results of feature selection because each and every method follows a unique method for identifying the important features. Hence the results from all these methods are comprehended in order to get better results. 


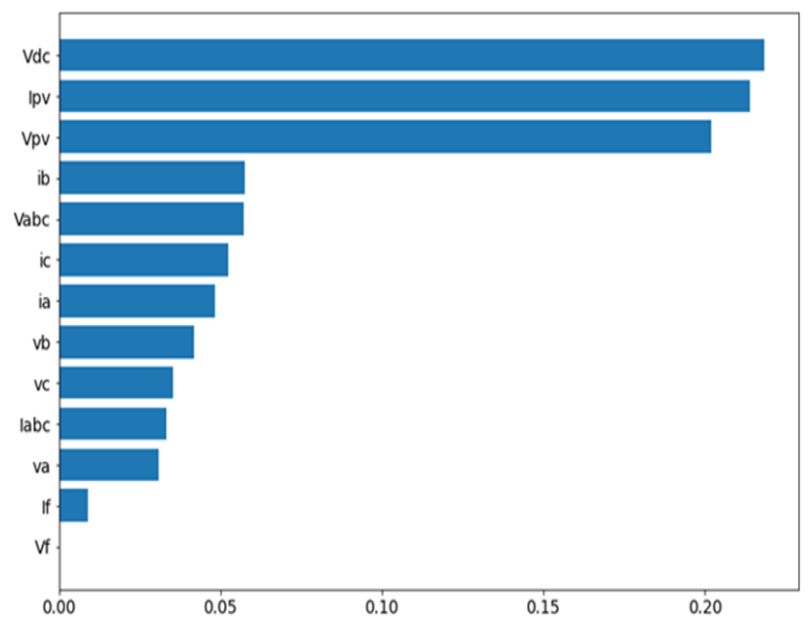

Fig. 2. Feature importance with extra tree classifier method

From the above figure, one can visualize the results of the feature importance method of feature selection with Extra tree classifier model. Here the different scores for each and every feature have been specified. Here the features with higher scores can be chosen as the important feature because the output class will be highly dependent on these features only. From the graph shown above Vdc, Ipv, Vpv, Ib, Vabc as the top five features chosen. Since the score of Vf is zero this clearly shows that it is highly irrelevant to classification.

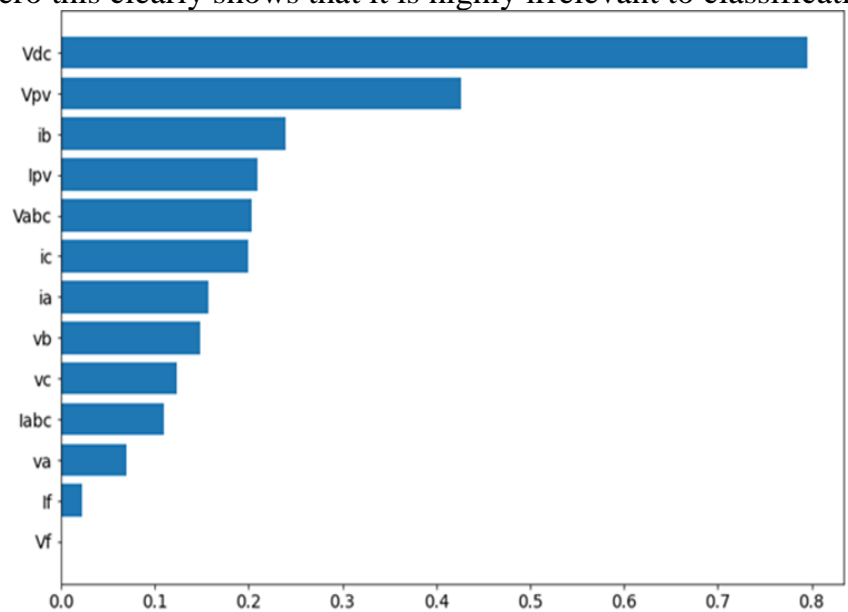

Fig. 3. Feature importance with permutation method

From fig 3, the output bar graph obtained with the scores of Feature importance method with Permutation importance as a model can be visualized. This clearly shows that the following features, Vdc, Vpv, Ib, Ipv, and Vabc can be chosen as an important feature. Though there is a slight variation in their order because of the difference in their identification method hence, the results obtained from these two different methods can be considered. 


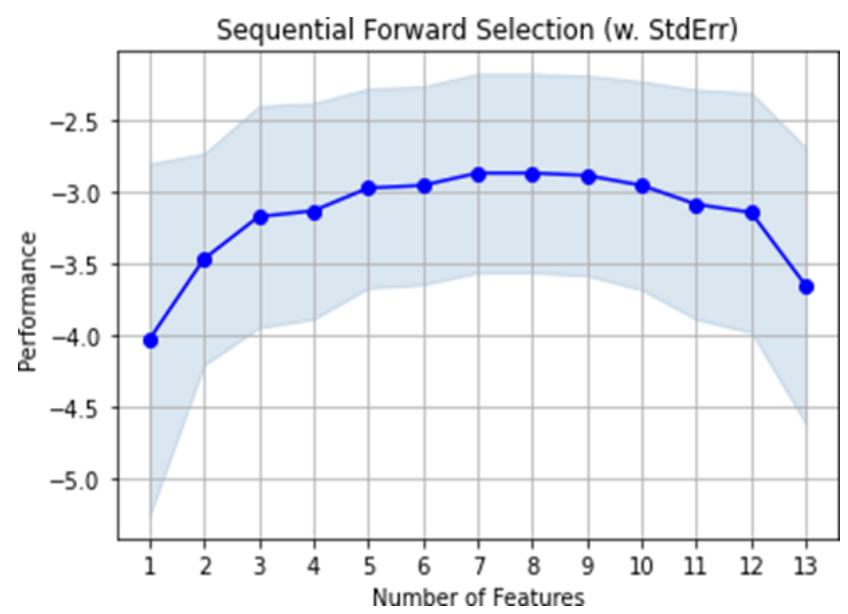

Fig. 4. Wrapper method with Sequential Forward selection

From fig 4, one can visualize the results of wrapper feature selection method with sequential forward selection. Theory related to this concept has been explained in the previous chapter. Here the output of the feature selector is obtained with the graph plotted against the feature and the performance value. The features are numbered according to the table I.

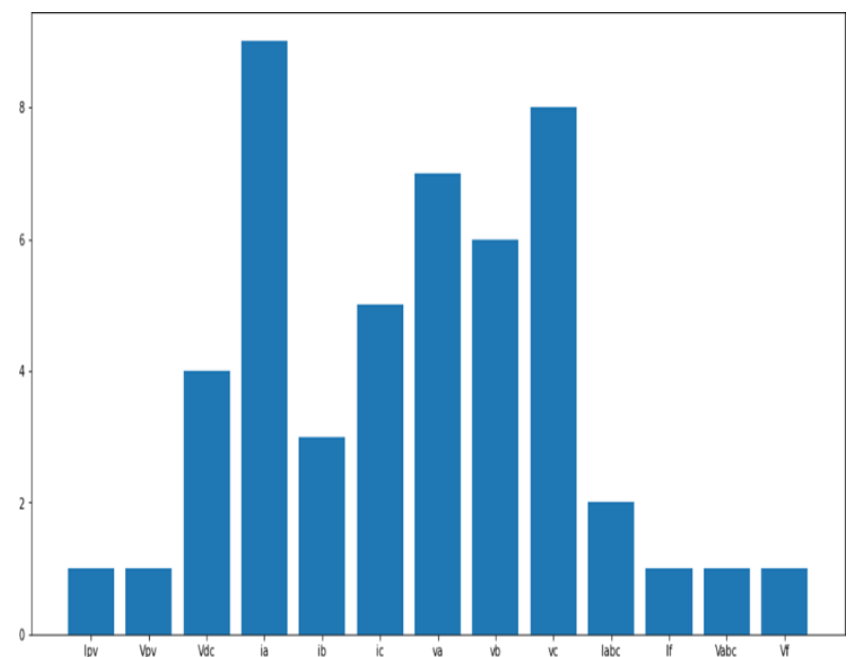

Fig. 5. Wrapper method with Reverse feature elimination

Fig 5, is the result of Reverse feature elimination. Here the Features in the dataset is plotted against the ranking obtained from training the model RFE i.e., Reverse feature elimination. The ranking of each and every feature is listed below. The features with highest ranking can be considered as the selected feature. 


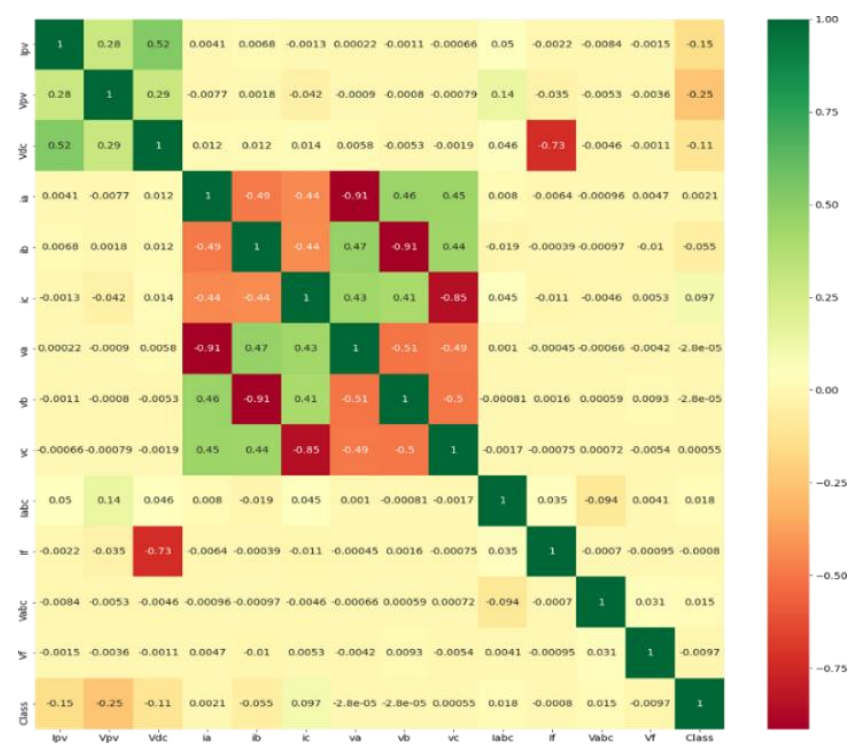

Fig. 6. Correlation matrix with heat map

From Fig 6, one can visualize the output of correlation matrix with heat map. Here the chart at the left side indicates that dark green color will be highly positively correlated and dark green color will be highly negatively correlated. Here the feature in our dataset along with the output class will be present both in horizontal and vertical sides. Features which are highlycorrelated with the output class should not correlate with any other features. Such features can only be taken into consideration. Since the results for feature selection has already been obtained from the wrapper method and feature selection method, using correlation matrix one can just cross check whether the selected features are highly correlated with other chosen features or not. By doing so one can finally conclude that the five most important features are, Vdc, Ipv, Vpv, Ib, and Vabc. Thus, while training and testing the model remaining features can be dropped from the dataset.

Nä̈ve bayes classifier

After the feature selection, the training of classifier was the next step. Those features selected through feature selection were used in training these models. First classifier which was chosen to be implemented was Naïve Bayes classifier.

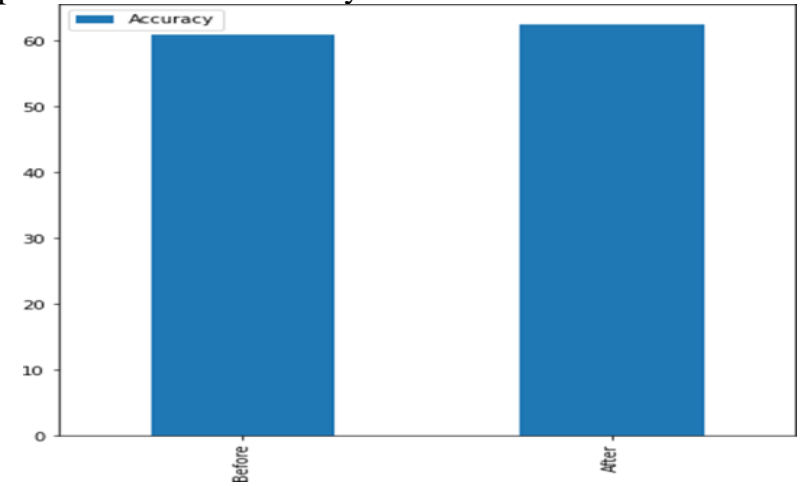

Fig. 7. Comparison of Naïve Bayes classifier before and after feature selection 
From Fig 7, the accuracy of the classifier with Naïve bayes model was around $60 \%$ without feature selection and with feature selection it was around 63\%. Reason behind the less accuracy is the nature of the dataset. Generally Naïve Bayes classifier works well for linear dataset, but here the data set was not linear and it was too large with decimal values. Hence the next classifier which was chosen to be implemented was One Vs Rest classifier.

One vs rest classifier

Here the obtained output after training and testing the classifier has been mentioned.

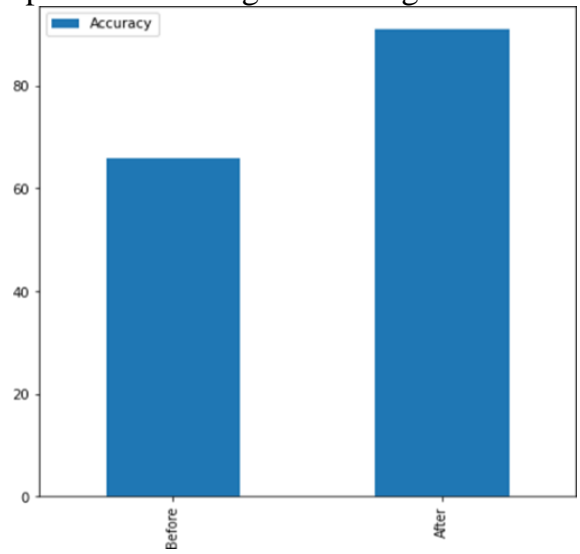

Fig. 8. Comparison of One Vs Rest classifier before and after feature selection

In the Fig 8, the comparison plot of One Vs Rest classifier with and without feature selection has been mentioned. While implementing OVR classifier it was observed that the accuracy was around $65 \%$ for the dataset without feature selection and it was around $91 \%$ for the dataset with feature selection. Unlike Naïve Bayes classifier this classifier produced a good result.

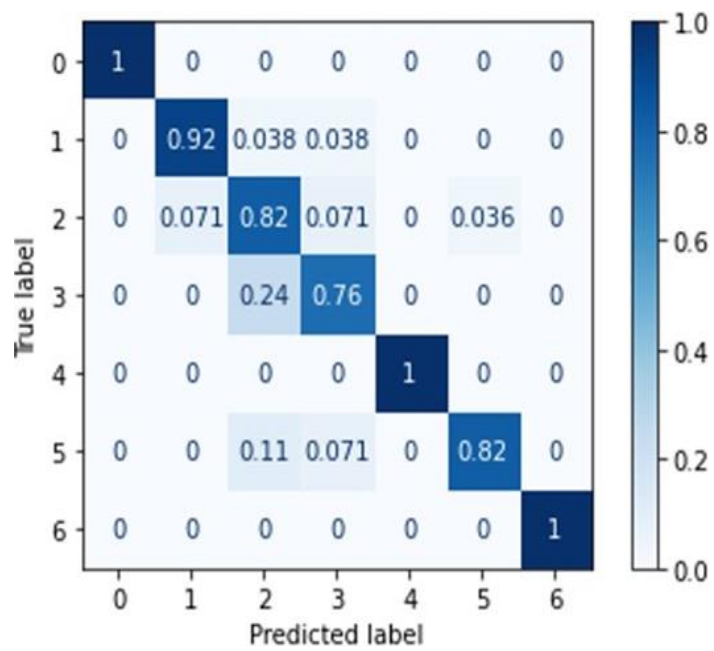

Fig. 9. Confusion matrix of One Vs Rest Classifier

Fig9, shows the confusion matrix of the OVR classifier. Confusion matrix is generally plotted with the Predicted label against the True label. Here from the color label, it is clearly visible that most of the classes are correctly predicted by the classifier. 


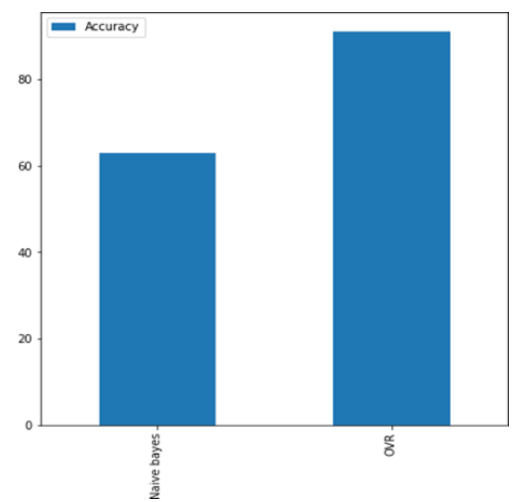

Fig. 10. Comparison of Naïve Bayes classifier with One Vs Rest

The Fig 10, is the comparison plot of the accuracy of Naïve Bayes and One Vs rest. As discussed earlier One Vs Rest classifier has a good performance with the accuracy of around $91 \%$.

Multilayer perceptron classifier

Multilayer Perceptron is a type of supervised machine learning model, which trains and test the data with the help of the activation function described in input, output and the hidden layers. As mentioned in the previous chapters the activation function plays a major role in deciding the performance of this model.

If the activation function is not chosen properly it might lead to few errors like over fitting. In order to overcome those issue each and every activation function has been implemented, and on comparing the results it is found that ReLUactivation functioned performed well for this particular classification problem. In Fig 13, one can clearly visualize a comparison between the different activation function tried to be implemented.

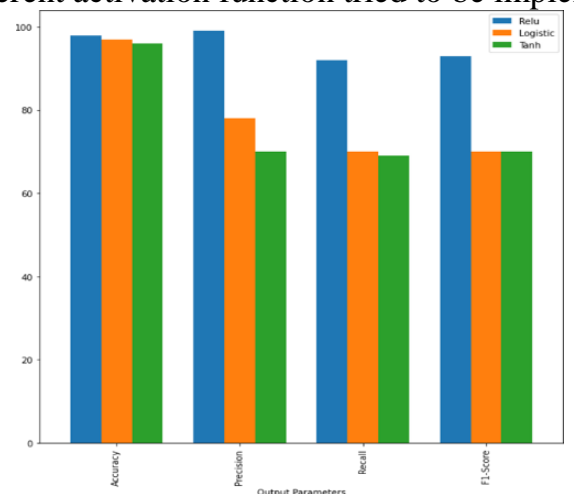

Fig. 11. Comparison of activation functions of MLP Classifier

From Fig 11, one can conclude that rectified linear unit activation function (ReLU) performs better than the other two activation function. Though there is only a slight variation in the value of accuracy, but the values of precision, recall and F1-score shows a huge variation due to over fitting.

Table I. Comparison of activation functions of MLP Classifier

\begin{tabular}{|l|l|l|l|l|l|}
\hline S.no & Activation function & Accuracy & Precision & Recall & F1-score \\
\hline 1 & ReLU & 98 & 99 & 92 & 93 \\
\hline 2 & Logistic & 97 & 78 & 70 & 70 \\
\hline 3 & Tanh & 96 & 70 & 69 & 70 \\
\hline
\end{tabular}


From Table I, one can identify the slight variation in the values of precision, recall and F1-score on using different activation function. As a result, it can be stated that the ReLU activation function outperforms both logistics and the Tanh function.

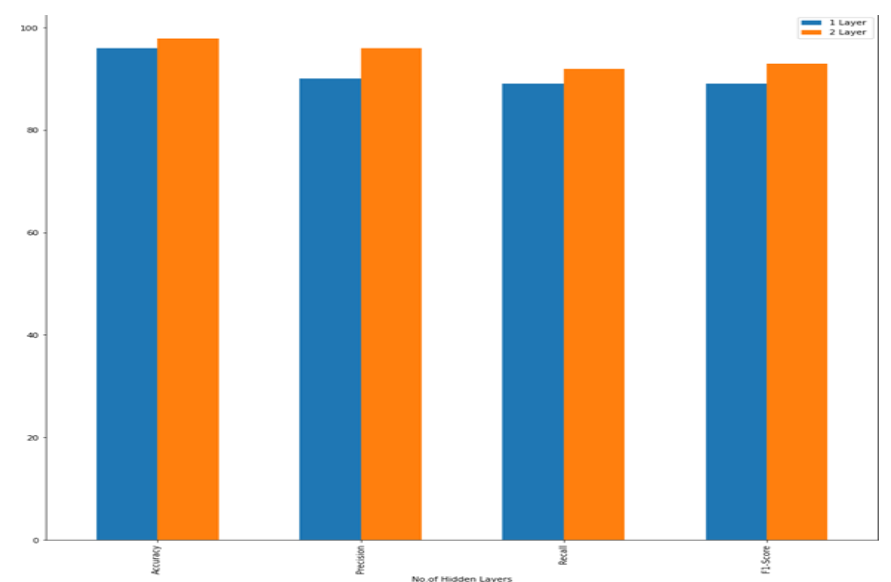

Fig. 12. Comparison of output parameters of different number of layers

Fig 12, is the plot obtained from the comparison of the output parameters of different number of hidden layers in the MLP Classifier with rectified linear activation function.

Table II. Comparison of output parameters of different number of layers

\begin{tabular}{|l|l|l|l|l|l|}
\hline S.No & No of Hidden layers & Accuracy & Precision & Recall & F1-score \\
\hline 1 & 1 & 96 & 90 & 89 & 89 \\
\hline 2 & 2 & 97.8 & 96 & 92 & 93 \\
\hline
\end{tabular}

From Table II, one can note that the performance of the classifier is best with the number of hidden layers as two. And here the architecture of the hidden layers was 6-6 where 6 is the number of nodes at each layer. Here the architecture of 6-6 is chosen because of the shape of the input features. Increase the number of features may lead to issues like over fitting which generally affects the performance of the classifier. The confusion matrix obtained from the MLP Classifier with 2 Hidden layers i.e. (6-6) is mentioned below. 


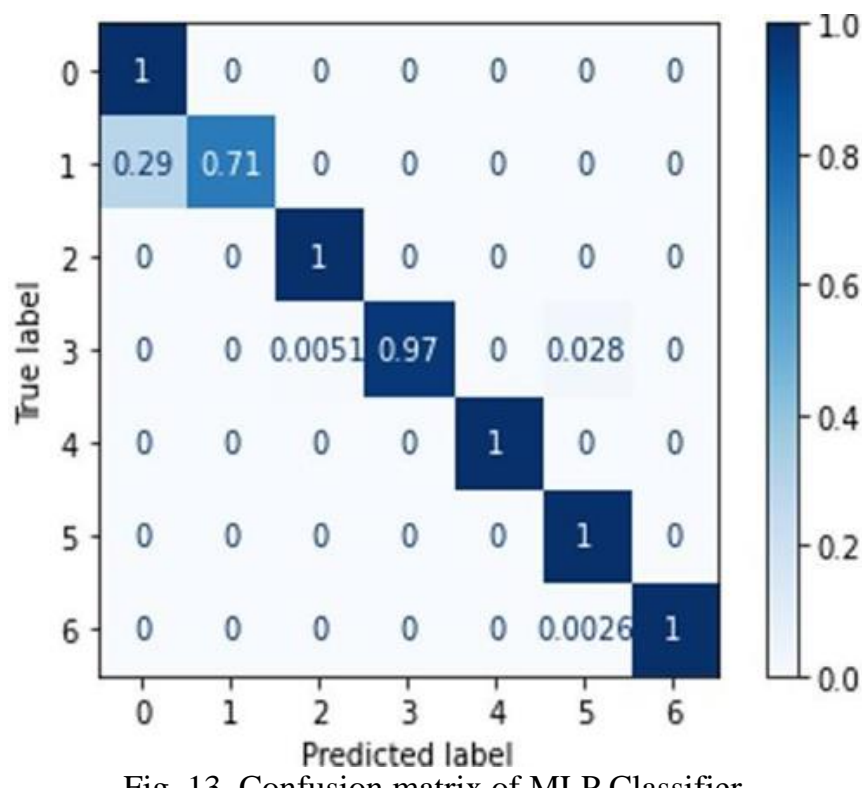

Fig. 13. Confusion matrix of MLP Classifier

In Fig 13, the confusion matrix obtained from the neural network model has been included. This shows a better confusion matrix than the One Vs rest classifier. Here the False positive and false negative values are too low because of the good accuracy of the model. The comprehensive results of all the classifier implemented will be discussed further.

Comparision of ovr, nä̈ve bayes and mlp classifier

A comparison on all the three classifiers will be discussed further. From Fig 14, the performance of all the three different classifiers can be visualized. The performance of Naive Bayes classifier was not highly perfect because of the nature of the dataset. But One Vs Rest classifier and Multi-Layer perceptron based Neural Network performed well for this particular application. On comparison with the output parameters of OVR and MLP there was only slight variations.

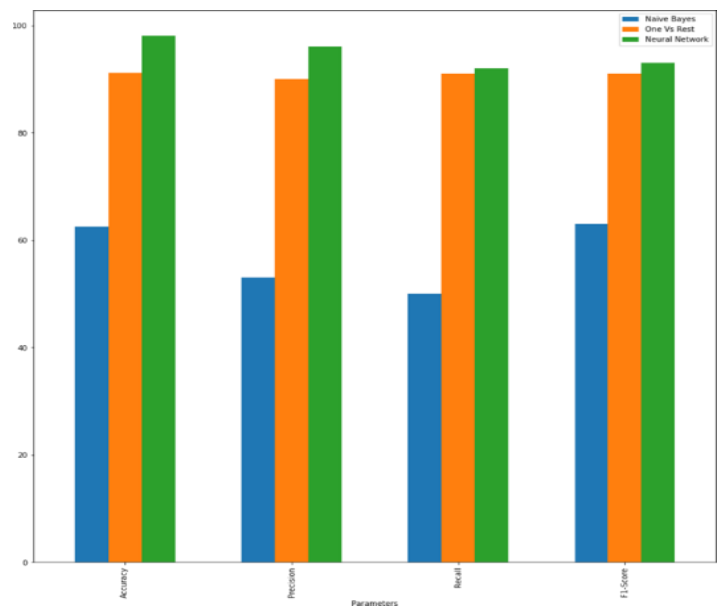

Fig. 14. Comparison of output parameters of OVR, Naïve bayes and MLP Classifier 
Table III. Comparison of Output Parameters of Naïve Bayes, OVR, and MLP Classifier

\begin{tabular}{|l|l|l|l|l|l|}
\hline S.no & Classifier & Accuracy & Precision & Recall & F1-Score \\
\hline 1 & Naïve Bayes & 62.5 & 53 & 50 & 63 \\
\hline 2 & One Vs Rest & 91.05 & 90 & 91 & 91 \\
\hline 3 & Multi-Layer Perceptron & 97.99 & 96 & 92 & 93 \\
\hline
\end{tabular}

From Table III, one can conclude that the Accuracy of MLP classifier was higher i.e., around $97 \%$ and the accuracy of One Vs Rest classifier was around 91\% which is slightly lesser than MLP Classifier. And the value of Precision was also good for both the classifier OVR and MLP i.e., around 90 and 96 respectively. The value of Recall was around 91 and 92 for OVR and MLP classifier, and finally the F1- score of OVR was around 91 and for MLP it was around 93.Thus, the neural network-based model had better results on comparison with other models, but the only drawback is the training time and testing time. If the time delay is not a major issue, one can go with MLP classifier or else One Vs Rest classifier will be of a better choice in that case.

\section{Conclusion And Future Work}

The identification and classification of faults that can occur in a Photo Voltaic system are quite essential for efficient generation of power. By employing Naïve Bayes, One Vs Rest and Multi-Layer Perceptron Classification algorithms the faults viz., Partial shading, Bypass diode, Bridging, temperature, Complete shading and Short circuit fault in a PV system were classified. By detecting the faults, one can prevent the chances of reduction in efficiency and therefore ensure proper generation of power in the Photo Voltaic systems. The proposed model was able to achieve accuracy of about $97 \%$ which is considerably higher. Therefore, the model can serve to predict and classify the faults in a PV system.

The prediction of the type of fault that may occur during PV Systems operation is successfully done by the proposed model with an accuracy of $97 \%$. However, work still needs to be done to achieve reproducibility, which can be verified by testing the model against various other datasets. Apart from the proposed model which uses Naïve Bayes, One Vs Rest and Multi-layer Perceptron classifier more models which makes use of other classification algorithms such as KNN, SVM can also be developed. Thus, the results from these algorithms can be compared with the existing model to select the better performing model. Apart from simulation, the model has to be tested in real-time. To achieve this, the model has to be deployed in the cloud, and a control system which employs a microcontroller can be developed to test with actual physical quantities. Such an IOT related setup can be physically made and the data can be processed for further classification. The results can then be used to further improve the model. Furthermore, the model should also be tested against various noises that may occur in the real world. 


\section{References}

[1] Elyes Garoudjaa, AissaChouderb, Kamel Karaa, and Santiago Silvestre, "An enhanced machine learning based approach for failures detection and diagnosis of PV systems," Energy Conversion and Management, Vol 151, pp.496-513, 2017.

[2] Ye Zhao, Ling Yang, Brad Lehman, Jerry Mosesian, Jean-Francois de Palma and Robert Lyons, "Decision tree-based fault detection and classification in solar photovoltaic arrays," TwentySeventh Annual IEEE Applied Power Electronics Conference and Exposition (APEC), pp. 93-99, 2012.

[3] K. Pahwa, M. Sharma, M. S. Saggu and A. Kumar Mandpura, "Performance Evaluation of Machine Learning Techniques for Fault Detection and Classification in PV Array Systems," International conference of Signal processing and Integrated networks, pp. 791-796,2020.

[4] Dhanup S. Pillai, and N. Rajasekar, "A Comprehensive review on protection challenges and fault diagnosis in PV systems," Renewable and Sustainable Energy Reviews, Vol 91, pp. 18-40, 2018.

[5] M. Mano Raja Paul, R. Mahalakshmi and R. Jai Ganesh, "Classification and Detection of Faults in Grid Connected PV Systems,” International Journal of Scientific \& Engineering Research, Vol 7, pp $147-154,2016$.

[6] M.G. Bello, "Enhanced training algorithms, and integrated training/architecture selection for multilayer perceptron networks," IEEE Transactions on Neural Networks, Vol 3, pp.864-875, 1992.

[7] "Basics of Photovoltaic Systems, its Components and Working" [Online] Available: https://energyeducation.ca/encyclopedia/Photovoltaic_system

[8] "Naive Bayes for Machine Learning" [Online] Available: https://machinelearningmastery.com/naive-bayes-for-machine-learning/

[9] "One-Vs-Rest strategy for multiclass classification" [Online] Available: https://www.geeksforgeeks.org/one-vs-rest-strategy-for-multi-class-classification/

[10] "Introduction to Neural Networks, Advantages and Applications" [Online] Available: https://towardsdatascience.com/introduction-to-neural-networks-advantages/

[11] "Grid Connected Photovoltaic Systems - Dataset" [Online]Available: 